\title{
Patterns of MHC-DRB1 polymorphism in a post-glacial island canid, the Newfoundland red fox (Vulpes vulpes deletrix), suggest balancing selection at species and population timescales
}

\author{
H. Dawn Marshall ${ }^{1}$ • Barbara L. Langille ${ }^{1}$ - Crystal A. Hann ${ }^{1} \cdot$ Hugh G. Whitney ${ }^{2}$
}

Received: 5 November 2015 / Accepted: 4 February 2016/Published online: 19 February 2016

(C) The Author(s) 2016. This article is published with open access at Springerlink.com

\begin{abstract}
As the only native insular Newfoundland canid between the extinction of the wolf in the 1930s and the recent arrival of coyotes, the red fox (Vulpes vulpes deletrix Bangs 1898) poses interesting questions about genetic distinctiveness and the post-glacial colonization history of the island's depauperate mammalian fauna. Here, we characterized genetic variability at the major histocompatibility complex (MHC) class II DR $\beta 1$ domain (DRB1) locus in 28 red foxes from six sampling localities island-wide and compared it with mitochondrial control region (CR) diversity and DRB1 diversity in other canids. Our goals were to describe novel DRB1 alleles in a new canid population and to make inferences about the role of selection in maintaining their diversity. As in numerous studies of vertebrates, we found an order-of-magnitude higher nucleotide diversity at the DRB1 locus compared with the CR and significantly positive nonsynonymous-to-synonymous substitution ratios, indicative of selection in the distant past. Although the evidence is weaker, the Ewens-Watterson test of neutrality and the geographical distribution of variation compared with the CR suggest a role for selection over the evolutionary timescale of populations. We report the first genetic data from the DRB1 locus in the red fox and establish baseline information regarding immunogenetic variation in this island canid population which should inform continued
\end{abstract}

H. Dawn Marshall

dawnm@mun.ca

1 Department of Biology, Memorial University of Newfoundland, St. John's, Newfoundland and Labrador A1B 3X9, Canada

2 Animal Health Division, Forestry and Agrifoods Agency, Box 7400, St. John's, Newfoundland A1E 3Y5, Canada investigations of population demography, adaptive genetic diversity, and wildlife disease in red foxes and related species.

Keywords Red fox · Major histocompatibility complex · DRB1 $\cdot$ Balancing selection $\cdot$ Tests of neutrality $\cdot$ Host immunogenetics

\section{Introduction}

The major histocompatibility complex (MHC) contains among the most polymorphic genes in the vertebrate genome (Garrigan and Hedrick 2003). Allele frequencies (Hedrick and Thomson 1983), nucleotide sequence variation (Hughes and Nei 1988), linkage disequilibrium (Klitz and Thomson 1987), and allele genealogies (Takahata 1990) of many MHC genes are thought to be largely patterned by balancing selection, although this is a paradigm which is neither fully explanatory (Garrigan and Hedrick 2003; van Oosterhout 2009) nor is the intensity of selection estimated necessarily high (Satta et al. 1994; Yasukochi and Satta 2013). Nonetheless, in many species, variation in these prominent immune system genes is associated with disease agent pathogenicity, resistance, or susceptibility (e.g., Wassom et al. 1983; Buitkamp et al. 1996; Paterson et al. 1998; Grimholt et al. 2003; McClelland et al. 2003; Biedrzycka et al. 2011; Tsai et al. 2013). As such, the role of host genetic diversity in spread of infectious disease remains an open question in disease ecology, necessitating information from a wide variety of non-model wildlife organisms (Lively et al. 2014). From both evolutionary and ecological perspectives then, MHC genes provide a window into the study of functionally relevant adaptive genetic variation.

MHC class II genes, expressed on antigen-presenting cells, recognize extracellular pathogens such as bacteria and nematodes (Hughes and Yeager 1998). The class II gene series DP, 
DQ, and DR encode heterodimeric proteins each with $\alpha$ and $\beta$ chains. The DR $\beta 1$ domain (DRB1) gene, especially exon 2 , has been extensively studied in mammals, in part because it encodes functionally important antigen binding sites and in part because tight linkage to other class II genes allows variability of the DRB to predict variability at other loci (reviewed in Sommer 2005). For a non-model organism such as the red fox, the DRB1 exon 2 thus provides a practical starting point to investigate functional polymorphism within the MHC.

The island of Newfoundland, part of the Canadian province of Newfoundland and Labrador and North America's easternmost land mass, is host to only 14 indigenous terrestrial mammal species in four orders, including seven in Carnivora. Most of this fauna colonized the island post-glacially, within the last 7000 years (South 1983). Widely distributed in North America, Eurasia, northern Africa, and Australia, the red fox (Vulpes vulpes L.) comprises some 40 subspecies and occupies a broad range of habitats from tundra to urban (Larivière and Pasitschniak-Arts 1996). The Newfoundland red fox ( $V$. vulpes deletrix Bangs 1898), described as pale and straw-colored relative to mainland counterparts, has been considered distinct enough from neighboring mainland populations to warrant subspecies status (Dodds 1983). Newfoundland's red fox population is of special interest because insular Newfoundland is the only endemic focus in North America of the endoparasitic nematode French heartworm (Angiostrongylus vasorum), the causative agent of verminous pneumonia and coagulopathy (Jeffery et al. 2004). This parasite is of concern due to the possibility of transmission to domestic canines and the potential for spread to other parts of North America (Conboy 2011). In addition to wildlife diseases, the rapidly expanding coyote population in Newfoundland (McGrath et al. 2010) may threaten this island population of red foxes.

While studies of MHC variability tend to focus on species of conservation interest that have experienced population declines (e.g., Bowen et al. 2004; Babik et al. 2005; Huchard et al. 2006), Castillo et al. (2010) investigated the relationship between MHC diversity and the spread and maintenance of disease in a widespread and plentiful species, the North American raccoon (Procyon lotor), thus providing a comparative model for understanding adaptive genetic variation in non-threatened populations, as advocated by Kohn et al. (2006). Similar to raccoons, red foxes in Newfoundland present an excellent model for investigation of MHC variability in a disease-susceptible population experiencing novel challenges. Additionally, comparison of diversity at functional loci such as MHC and neutral ones such as the mitochondrial control region challenges the long-held tenet of conservation genetics that a relationship exists between levels of neutral and adaptive variation (Kohn et al. 2006). For example, a study of the MHC class II DRB and DQB loci in the San Nicolas Island fox (Urocyon littoralis dickeyi), one of the most monomorphic sexually reproducing animal populations known, revealed remarkably high levels of MHC variation (Aguilar et al. 2004).

The family Canidae in the mammalian Order Carnivora includes the closely related Canis species (dogs, wolves, and coyotes) and the progressively more distantly related South American foxes (genus Dusicyon), red fox-like canids (genus Vulpes), and grey and island foxes (genus Urocyon). Suites of DRB1 alleles have been described in several wild canid species including island foxes (Aguilar et al. 2004), Mexican grey wolves (Hedrick et al. 2000, 2003), red wolves and coyotes (Hedrick et al. 2002), African wild dogs (Marsden et al. 2009), and arctic foxes (Ploshnitsa et al. 2011). Our objectives here were to (1) characterize DRB1 alleles in the Newfoundland population of red foxes; (2) compare patterns of variation to mitochondrial control region (CR) variation from the same localities and DRB1 sequences from other canids; and in so doing (3) use a series of tests to evaluate the evidence for balancing selection in the current generation, on a population scale, and in the distant past. Ultimately, we aim to report the first data on DRB1 diversity in Newfoundland red foxes and establish a baseline for comparison for future studies investigating host genetic components of immunity in the Newfoundland red fox.

\section{Materials and methods}

\section{Study area and sample collection}

Salivary glands or muscle tissue from four to five red foxes from each of six sampling regions (Avalon Peninsula, Central, Northeast Coast, Northern Peninsula, West Coast, and South Coast) covering the geographic range of insular Newfoundland (28 in total; Fig. 1 and Table 1) was collected during 2002-2004 by the Animal Health Division of the Government of Newfoundland and Labrador, as part of an insular rabies eradication program, and during 2009-2012 by wildlife trappers from the Salmonier Nature Park. Animal tissue use was approved by the Institutional Animal Care Committee of Memorial University, in accordance with Canadian Council on Animal Care guidelines.

\section{Laboratory procedures}

DNA was extracted from salivary gland tissues using a QIAamp ${ }^{\circledR}$ DNA Mini kit (Qiagen Inc., Toronto, Canada) according to the manufacturer's instructions for tissue samples.

Exon 2 of the DRB1 locus (302 bp) was amplified with DRBIN1-F (5'-CCGTCCCCACAGCACATTTC-3') (Aguilar et al. 2004) and HDMDRB-R (5'CAGGCGCCCGCTGCGCTCAC-3'), modified from Aguilar et al. (2004). PCRs were performed in a final reaction volume of $25 \mu \mathrm{L}$ containing $1 \times$ Qiagen PCR 


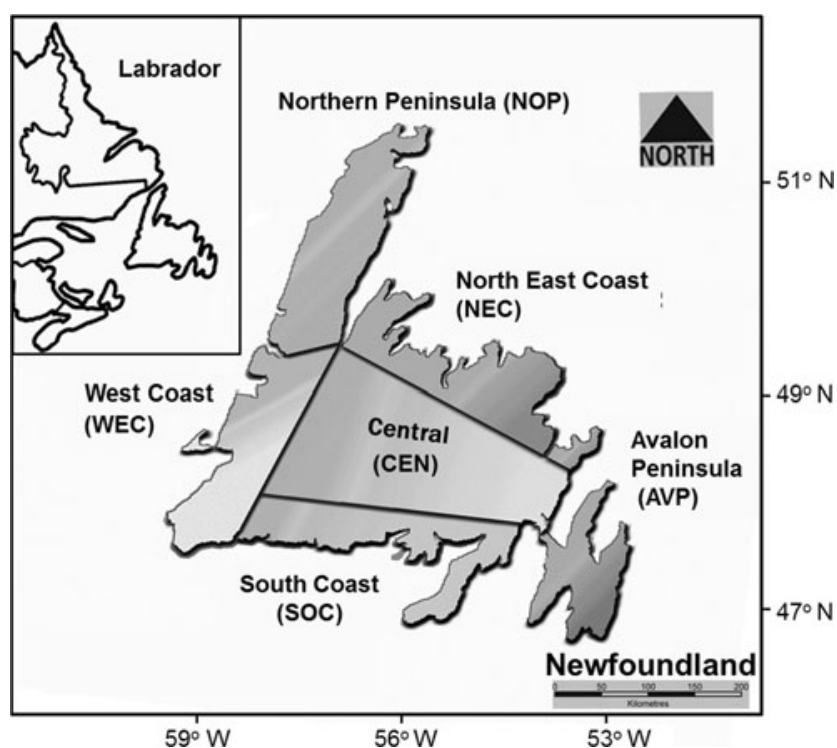

Fig. 1 Geographic location of the six sampled island Newfoundland red fox populations (Northeast Coast, Central Newfoundland, Avalon Peninsula, West Coast, South Coast, Northern Peninsula)

buffer, $200 \mu \mathrm{M}$ dNTPs (New England Biolabs Inc., Whitby, Canada), $400 \mathrm{nM}$ each primer, $1 \mathrm{U}$ HotStar Taq DNA polymerase (Qiagen Inc.), and 25-200 ng DNA template. The amplification profile for DRB1 consisted of an initial denaturation for $5 \mathrm{~min}$ at $95{ }^{\circ} \mathrm{C}$, followed by 14 cycles of $95{ }^{\circ} \mathrm{C}$ for $30 \mathrm{~s}, 62{ }^{\circ} \mathrm{C}$ (decreasing by $0.5{ }^{\circ} \mathrm{C}$ per cycle) for $30 \mathrm{~s}$, and $72{ }^{\circ} \mathrm{C}$ for $1 \mathrm{~min}$, followed by 25 cycles of $95{ }^{\circ} \mathrm{C}$ for $30 \mathrm{~s}, 55^{\circ} \mathrm{C}$ for $30 \mathrm{~s}$, and $72{ }^{\circ} \mathrm{C}$ for $1 \mathrm{~min}$, and a final $10-\mathrm{min}$ elongation at $72{ }^{\circ} \mathrm{C}$. PCR products were purified using the QIAquick PCR Purification kit (Qiagen Inc.).

DRB1 amplicons were then cloned using the Qiagen PCR Plus Cloning kit (Qiagen Inc.) following the manufacturer's instructions. Inserts were directly amplified from five to ten colonies for each amplicon using the M13 primers recommended by Qiagen, with reaction mixtures prepared as above but using a small portion of the bacterial colony as a template, and an amplification profile consisting of initial denaturation for $5 \mathrm{~min}$ at $95{ }^{\circ} \mathrm{C}$, followed by 40 cycles of $94{ }^{\circ} \mathrm{C}$ for $30 \mathrm{~s}$, $50{ }^{\circ} \mathrm{C}$ for $30 \mathrm{~s}$, and $72{ }^{\circ} \mathrm{C}$ for $2 \mathrm{~min}$, and a final extension at $72{ }^{\circ} \mathrm{C}$ for $10 \mathrm{~min}$. PCR products were purified as above.

All PCR products were sequenced in both directions using BigDye Terminator 2.0 chemistry and electrophoresed on the ABI Prism 3130 DNA Analyzer (Applied Biosystems Inc., Foster City, USA) using Sequencing Analysis version 5.0 software. Sequence reads were assembled and edited with Sequencher version 4.9 (Gene Codes Corporation, Ann Arbor, USA).

Initial examination of MHC DRB1 exon 2 sequences identified as many as eight distinct sequences per individual fox. However, molecular cloning produces errors such as recombinant alleles, singleton mutations, and non-target DNA
Table 1 DRB1 allele and genotype frequencies among six Newfoundland island samples of red foxes

\begin{tabular}{|c|c|c|c|c|c|c|c|}
\hline \multirow[t]{2}{*}{ Allele } & \multicolumn{7}{|c|}{ Locality } \\
\hline & NOP & WEC & NEC & CEN & SOC & AVP & All \\
\hline $2 \mathrm{~N}$ & 10 & 8 & 10 & 8 & 10 & 10 & 56 \\
\hline Vuvu DRB1 & 2 & 5 & 1 & 1 & 3 & 2 & 14 \\
\hline Vuvu DRB2 & 3 & & 2 & 4 & 2 & 3 & 14 \\
\hline Vuvu DRB3 & 2 & 1 & 4 & 1 & 1 & 2 & 11 \\
\hline Vuvu DRB4 & 1 & & 1 & & 2 & 1 & 5 \\
\hline Vuvu DRB5 & & 1 & & 1 & & 2 & 4 \\
\hline Vuvu DRB6 & 2 & & & & 1 & & 3 \\
\hline Vuvu DRB7 & & 1 & 1 & & 1 & & 3 \\
\hline Vuvu DRB8 & & & 1 & 1 & & & 2 \\
\hline \multicolumn{8}{|l|}{ Genotype } \\
\hline$N$ & 5 & 4 & 5 & 4 & 5 & 5 & 28 \\
\hline $2 / 3$ & 1 & & 1 & 1 & & 1 & 4 \\
\hline $1 / 1$ & & 2 & & & 1 & & 3 \\
\hline $1 / 2$ & 2 & & & & 1 & & 3 \\
\hline $2 / 4$ & & & 1 & & 1 & 1 & 3 \\
\hline $1 / 5$ & & 1 & & & & 1 & 2 \\
\hline $1 / 8$ & & & 1 & 1 & & & 2 \\
\hline $3 / 6$ & 1 & & & & 1 & & 2 \\
\hline $3 / 7$ & & 1 & 1 & & & & 2 \\
\hline $2 / 5$ & & & & 1 & & 1 & 2 \\
\hline $1 / 3$ & & & & & & 1 & 1 \\
\hline $3 / 3$ & & & 1 & & & & 1 \\
\hline $2 / 2$ & & & & 1 & & & 1 \\
\hline $4 / 6$ & 1 & & & & & & 1 \\
\hline $4 / 7$ & & & & & 1 & & 1 \\
\hline
\end{tabular}

For genotype designations, a shortened version of the allele name (one for Vuvu DRB1, etc.) is used. DRB1 alleles are described in Fig. 2

$N O P$ Northern Peninsula, WEC West Coast, $N E C$ North-East Coast, $C E N$ Central, SOC South Coast, AVP Avalon Peninsula

incorporation into the final sequence (Saitoh and Chen 2008). Following Castillo et al. (2010), to avoid such errors, distinct sequences were only considered alleles if they were not obvious recombinants of other alleles in the individual and if they differed from the other alleles in the individual by at least two nucleotide substitutions. Additionally, DRB PCR products from the 28 foxes were independently amplified and directly sequenced, and the two alleles per fox were verified only if they were observed as one of the cloned sequences inserts and could be also "phased" from the PCR product direct sequence.

\section{Characterization of DRB1 allele diversity in Newfoundland red foxes}

Variable sites among alleles were identified and annotated with the aid of MEGA version 6 (Tamura et al. 2013), and 
descriptive measures of diversity (number of alleles, expected heterozygosity, nucleotide diversity, and synonymous and nonsynonymous distances), in each location and overall, were calculated using MEGA or Arlequin version 3.5 (Excoffier et al. 2007).

\section{Tests of selection}

The following tests were performed as suggested by Garrigan and Hedrick (2003). To test for selection in the current generation, observed and expected heterozygosities (as calculated using ARLEQUIN) in each sampling location and overall were compared with chi-square tests for fit to HardyWeinberg expectations. Selection over the history of populations was evaluated by (1) the Ewens-Watterson test of neutrality, conducted using the software PyPop (Lancaster et al. 2003) over the entire sample of alleles - this test was also conducted for CR haplotypes from Newfoundland red foxes across the same sampling localities (Langille et al. 2014) to help dissect the effects of demography versus balancing selection on the outcome of this test, and (2) patterns of geographic variation-conventional $F_{\mathrm{ST}}$ (based on haplotype frequencies) and $d_{\mathrm{A}}$ (net number of nucleotide differences between populations; Nei and Li 1979) measures of population differentiation were calculated among each pair of sampling localities for both DRB1 data and compared to similar results from the CR data using a Mantel test, implemented in ARLEQUIN. Selection in the distant past was surveyed with (1) codonbased $Z$ tests of neutrality, which test for $\mathrm{dN}-\mathrm{dS}$ values different from 0 and also site-specific tests of $\mathrm{dN}-\mathrm{dS}$ using the HyPhy option, as conducted in MEGA, and (2) relationships among red fox DRB1 alleles and two sets of alleles representing other canids (Vulpes lagopus only and V. lagopus, U. littoralis, and Canis lupus) accessed from GenBank were determined via neighbor-joining phylogenies of maximum composite likelihood distances and tested by 10 , 000 bootstrap replicates, using MEGA, as a measure of transspecies polymorphism.

\section{Results and discussion}

Herein, we describe and characterize class II MHC DRB1 alleles in red foxes ( $V$. vulpes), from the island of Newfoundland, Canada. Our goals were (1) to make inferences about the role of balancing selection over short, medium, and longer term evolutionary scales in maintaining patterns of allelic diversity at this locus in Newfoundland red foxes and (2) to introduce data regarding diversity at a functional immune system locus as a baseline for future studies in this wildlife population. We present evidence in favor of balancing selection over the medium and especially longer evolutionary timescales in this species and compare patterns of diversity in Newfoundland foxes with those observed for other canids.

\section{Red fox DRB1 allelic diversity}

After removal of recombinants, cloned sequences that differed by only one nucleotide and any cloned sequence that could not be confirmed by direct sequencing of the PCR product, eight distinct alleles representing a 262-bp portion of the DRB1 exon 2 were identified among the 28 red foxes (deposited in GenBank with Accession numbers KU519427-KU519434) and each fox was found to contain at most two alleles, consistent with a single locus. Allele and genotype frequencies in each of the six Newfoundland red fox localities are given in Table 1 . Three common alleles accounted for $~ 70 \%$ of the 56 alleles sampled: Vuvu DRB1 (25\%), Vuvu DRB2 (25\%), and Vuvu DRB3 (19.6\%). Most (23/28) foxes were heterozygotes.

Among the eight alleles, 38 nucleotide sites were variable (Fig. 2a). The vast majority of these were at first or second positions of the codon, corresponding to 20 variable amino acid sites (Fig. 2b). Alleles differed from each other by 3-26 nucleotides or 2-17 amino acids. Phylogenetic relationships among the red fox alleles are shown in Fig. 3a. While allele Vuvu DRB2 is quite distinct from the others, the only wellsupported grouping of alleles are Vuvu DRB3 with Vuvu DRB7 (86 \%) and Vuvu DRB4 with Vuvu DRB8 (77 \%).

Nucleotide diversity for the DRB1 locus ( $\pi=0.030-0.061$; Table 2) is an order of magnitude higher than the CR $(\pi=0.0017-0.0062$; Langille et al. 2014) and is fairly consistent across localities, although lower in the West Coast (WEC) sample. On the other hand, expected heterozygosity was in the range $H_{\mathrm{E}}=0.56-0.82$ and very similar to the haplotypic diversity of $h=0.54-0.69$ for the CR (Langille et al. 2014), despite a greater number of alleles relative to sample size (eight in $2 \mathrm{~N}=56$ vs seven in $N=159$ ). Patterns of variability at the DRB1 locus among Newfoundland red foxes locations are uncorrelated to variability at the $\mathrm{CR}\left(R^{2}=0.203\right.$ for nucleotide diversity and 0.076 for expected heterozygosity/haplotypic diversity; $P>0.05$ ).

\section{Selection at the timescales of species and populations but not in the current generation}

Expected and observed measures of heterozygosity for each location and overall are presented in Table 2. There were no departures from Hardy-Weinberg expectations in any location or overall $(P \gg 0.05)$, indicating no evidence of selection in the short term. Although the small sample size investigated here may compromise power for this test, the observed and expected heterozygosities are exactly the same, so there is certainly no indication that a departure from Hardy-Weinberg equilibrium is present. 
Fig. 2 Variable sites in eight DRB1 alleles identified among 28 red foxes from six Newfoundland island localities. Alleles are designated Vuvu DRB1-8, in order of frequency of occurrence. a Thirty-seven variable nucleotide sites, grouped according to codon membership. Second position sites are shown in bold; third position sites are italicized. b Twenty variable amino acid sites, aligned with respect to the appropriate nucleotide codon

Allele

a

$\begin{array}{ll}\text { Vuvu } & \text { DRB1 } \\ \text { Vuvu } & \text { DRB2 } \\ \text { Vuvu } & \text { DRB3 } \\ \text { Vuvu } & \text { DRB4 } \\ \text { Vuvu } & \text { DRB5 } \\ \text { Vuvu } & \text { DRB6 } \\ \text { Vuvu } & \text { DRB7 } \\ \text { Vuvu } & \text { DRB8 }\end{array}$

b

Vuvu DRB1

Vuvu DRB2

Vuvu DRB3

Vuvu DRB4

Vuvu DRB5

Vuvu DRB6

Vuvu DRB7

Vuvu DRB8
Nucleotide or Codon Position

$\begin{array}{lllllllllllll}11 & 1 & 1 & 1 & 11 & 11 & 1 & 12 & 2 & 2 & 22 & 22\end{array}$

$\begin{array}{lllllllllllllllllllllll}111 & 11 & 4 & 555 & 666 & 6 & 7 & 8 & 44 & 5 & 6 & 7 & 888 & 99 & 9 & 90 & 1 & 2 & 33 & 44\end{array}$ $\begin{array}{lllllllllllllllllllll}78 & 012 & 67 & 7 & 567 & 123 & 7 & 3 & 8 & 89 & 8 & 6 & 8 & 789 & 01 & 8 & 90 & 2 & 9 & 567 & 78\end{array}$ CA TTA GC G TAC GAA T C A GA A G C GAC GC A GC T G GGC CT AT GTA GG G CTG ATT G T C GA A G C CGC CG C GA A C TTT AT CA GGA GC G TTC GAA T C T GA A C C GAG GA A GC T G ATT AT AT GCA TC G TTC GAC T C A GA A G A GAC GC A GC T C TTT AC CA GGA GC A TTC GAA T C A GA A G C GAG AA C AA A G ATT AT AT TTT TC G CTC GAC T T T GA A G A GAC GC A GC T G GGC CT CA GGA GC G TTC GAA T C T AT C C C GAG GA A GC T G ATT AT CA TTA GC G TTC GAA T C A GA A G C GAC GC A GC T C TTT AC

\begin{tabular}{|c|c|c|c|c|c|c|c|c|c|c|c|c|c|c|c|c|c|c|c|}
\hline & & & 1 & 1 & 2 & 2 & 2 & 3 & $\begin{array}{l}5 \\
0\end{array}$ & $\begin{array}{l}5 \\
3\end{array}$ & 5 & 6 & 6 & 6 & 6 & 7 & $\begin{array}{l}7 \\
7\end{array}$ & 7 & $\begin{array}{l}8 \\
3\end{array}$ \\
\hline 3 & 4 & 6 & 6 & 9 & 1 & 3 & 5 & 0 & 0 & 3 & 6 & 0 & 3 & 4 & 7 & 1 & 7 & 9 & 3 \\
\hline$Q$ & $\mathrm{~L}$ & A & $\mathrm{R}$ & $\mathrm{Y}$ & $\mathrm{E}$ & $Y$ & $\mathrm{H}$ & $\mathrm{N}$ & D & $\mathrm{Y}$ & G & $\mathrm{L}$ & D & A & $\mathrm{A}$ & $\mathrm{F}$ & G & G & $\mathrm{L}$ \\
\hline M & V & G & $\mathrm{R}$ & $\mathrm{L}$ & I & D & $\mathrm{Y}$ & $\mathrm{H}$ & D & $\mathrm{Y}$ & G & $\mathrm{L}$ & $\mathrm{R}$ & $\mathrm{R}$ & $\mathrm{E}$ & $\mathrm{Y}$ & $\mathrm{R}$ & $\mathrm{F}$ & M \\
\hline$Q$ & G & A & $\mathrm{R}$ & $\mathrm{F}$ & $\mathrm{E}$ & $\mathrm{Y}$ & $\mathrm{H}$ & $\mathrm{Y}$ & D & $\mathrm{Y}$ & $\mathrm{R}$ & L & $\mathrm{E}$ & $\mathrm{E}$ & A & $\mathrm{F}$ & G & I & M \\
\hline$M$ & A & $\mathrm{S}$ & $\mathrm{R}$ & $\mathrm{F}$ & D & $Y$ & $\mathrm{H}$ & $\mathrm{N}$ & D & $\mathrm{Y}$ & $\mathrm{G}$ & I & $\mathrm{D}$ & A & A & $\mathrm{F}$ & $\mathrm{R}$ & $\mathrm{F}$ & 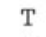 \\
\hline Q & G & A & Q & $\mathrm{F}$ & $\mathrm{E}$ & $\mathrm{Y}$ & $\mathrm{H}$ & $\mathrm{N}$ & D & $\mathrm{Y}$ & G & L & $\mathrm{E}$ & K & K & $\mathrm{Y}$ & G & I & M \\
\hline $\mathrm{M}$ & $\mathrm{F}$ & $\mathrm{S}$ & $\mathrm{R}$ & $\mathrm{L}$ & D & $Y$ & $\mathrm{Y}$ & $\mathrm{Y}$ & D & $\mathrm{Y}$ & $\mathrm{G}$ & I & D & A & $\mathrm{A}$ & $\mathrm{F}$ & G & G & L \\
\hline Q & G & A & $\mathrm{R}$ & $\mathrm{F}$ & $\mathrm{E}$ & $Y$ & $\mathrm{H}$ & $\mathrm{Y}$ & I & $\mathrm{S}$ & $\mathrm{R}$ & $\mathrm{L}$ & $\mathrm{E}$ & $\mathrm{E}$ & A & $\mathrm{F}$ & G & I & M \\
\hline Q & L & A & $\mathrm{R}$ & $\mathrm{F}$ & $\mathrm{E}$ & $\mathrm{Y}$ & $\mathrm{H}$ & $\mathrm{N}$ & D & $\mathrm{Y}$ & $\mathrm{G}$ & $\mathrm{L}$ & D & A & A & $\mathrm{F}$ & $\mathrm{R}$ & $\mathrm{F}$ & $\Gamma$ \\
\hline
\end{tabular}

Taken together, the patterns of allele frequency and geographic distribution between the red fox CR and DRB1 alleles are consistent with selection in the medium term. The results of the Ewens-Watterson test conducted with PyPop indicate marginal (Fnd $=-1.161 ; P=0.051$ ) evidence for departure from the expected allele frequency under neutrality for the 56 alleles at the DRB1 locus. Indeed when a simulation is done by doubling the sample size but keeping the allele distribution the same, significance is observed $(P=0.019)$. In contrast, the $\mathrm{CR}$ frequency distribution was quite consistent with neutrality (Fnd $=-0.461 ; P=0.396$ ), suggesting that the result in the DRB1 locus is not due to the effects of demography. Moreover, demographic tests of expansion for the CR imply no recent expansion of this population (Langille et al. 2014).

With respect to patterns of geographic variation inferred from the DRB1 data, there was no significant differentiation among six Newfoundland localities either derived from haplotype frequencies $\left(F_{\mathrm{ST}}=0.010 ; P=0.317\right)$ or pairwise differences $\left(d_{\mathrm{A}}=0.028 ; P=0.232\right)$. Pairwise estimates of $F_{\mathrm{ST}}$ or $d_{\mathrm{A}}$ among individual localities were significant only for the WEC population compared with the NEC and Central (CEN) localities (Table 3); in fact, only the WEC and CEN samples even showed positive levels of differentiation. This patterns contrasts with results for $\mathrm{CR}$ in which $F_{\mathrm{ST}}=0.0796$ and $d_{\mathrm{A}}=0.0599$ among all localities were significantly different from zero $(P \ll 0.05)$, and most pairwise values were positive (Langille et al. 2014). The DRB1 and CR matrices are not correlated for either $F_{\mathrm{ST}}(r=-0.099 ; P=0.572)$ or $d_{\mathrm{A}}$ $(r=-0.236 ; P=0.696)$. Lower $F_{\mathrm{ST}}$ values for the DRB1 compared with the CR may suggest uniform selection pressure across subpopulations (Garrigan and Hedrick 2003), although greater genetic drift of haploid loci may be a contributing factor.

MHC diversity in populations facilitates the immune response to various pathogens and parasites, contributing to disease resistance at the population level. That selection has played a role in DRB1 diversity in Newfoundland red foxes at the timescale of populations does receive support from the tests of selection performed here, although certainly caution is warranted regarding the strength of this conclusion. Nonetheless, it makes sense that over the $\sim 7000$-year period since re-colonization of Newfoundland, foxes and other species would experience novel exposure to disease and pathogens in their new environment. If particular alleles caused susceptibility or resistance to particular parasites then the frequency of those alleles may be expected to change over time. With the present data, it would be highly speculative to comment on this further with respect to particular parasites such as A. vasorum; however, future research effort involving documentation of association of specific alleles with disease status may be informative.

Significantly positive $Z$ tests of overall $\mathrm{dN} / \mathrm{dS}$ ratios were observed in Newfoundland red foxes, $V$. vulpes (Table 2). Perhaps more interesting, the HyPhy algorithm identified specific amino acid sites under significant positive selection (normalized $\mathrm{dN}-\mathrm{dS}>0 ; P<0.05)$ in a set of $V$. vulpes and $V$. lagopus sequences (Fig. $3 \mathrm{a}$ ) and in a more comprehensive set of canid sequences (Fig. 3b). In the former, one such site (64 in Fig. 2b) was identified. Three of the five amino acids at this site are found in alleles of both species, while the remaining two are species specific. Similarly, three sites $(30,56$, and 67 in Fig. 2b; site 64 was marginally significant at $P<0.10$ ) were found to be under positive selection in the inclusive data 
Fig. 3 Neighbor-joining phylogenies among red fox DRB1 alleles and DRB1 alleles from other canids accessed from GenBank. Accession numbers are given with taxon name, and allele designations are as stated in the accession. Numbers above nodes are percentage support from 10,000 bootstrap replicates. The blocks to the right show the amino acid associated with each taxon at selected sites identified by HyPhy, as discussed in the text. These correspond to sites 64 (a) and 30, 56, and 67 (b) in Fig. 2b. a Vulpes only phylogeny: Vula $=$ Vulpes lagopus and Vuvu DRB1Vuvu DRB8 designate the Vulpes vulpes alleles. b Vulpes, Urocyon, and Canis lupus comprehensive phylogeny. Urli = Urocyon littoralis and Calu = Canis lupus. Due to the very large number of alleles available from Canis sp., Canis lupus was selected here to represent the lineage a

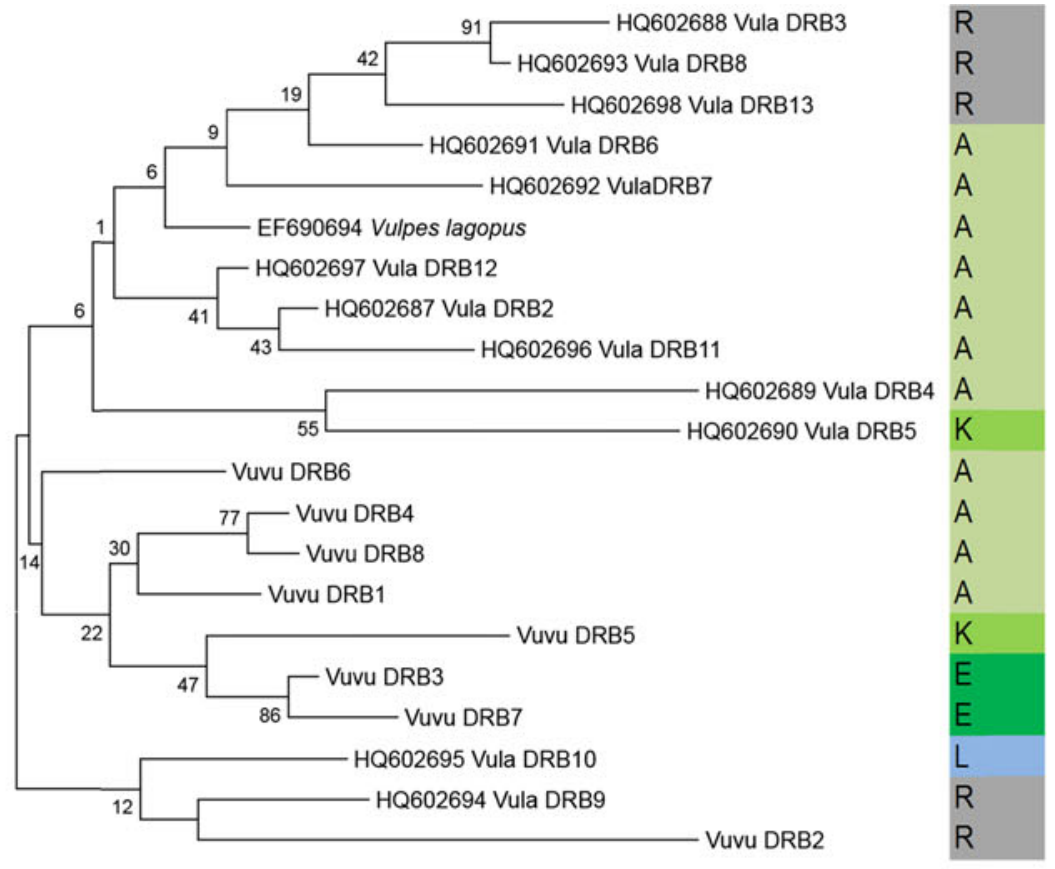

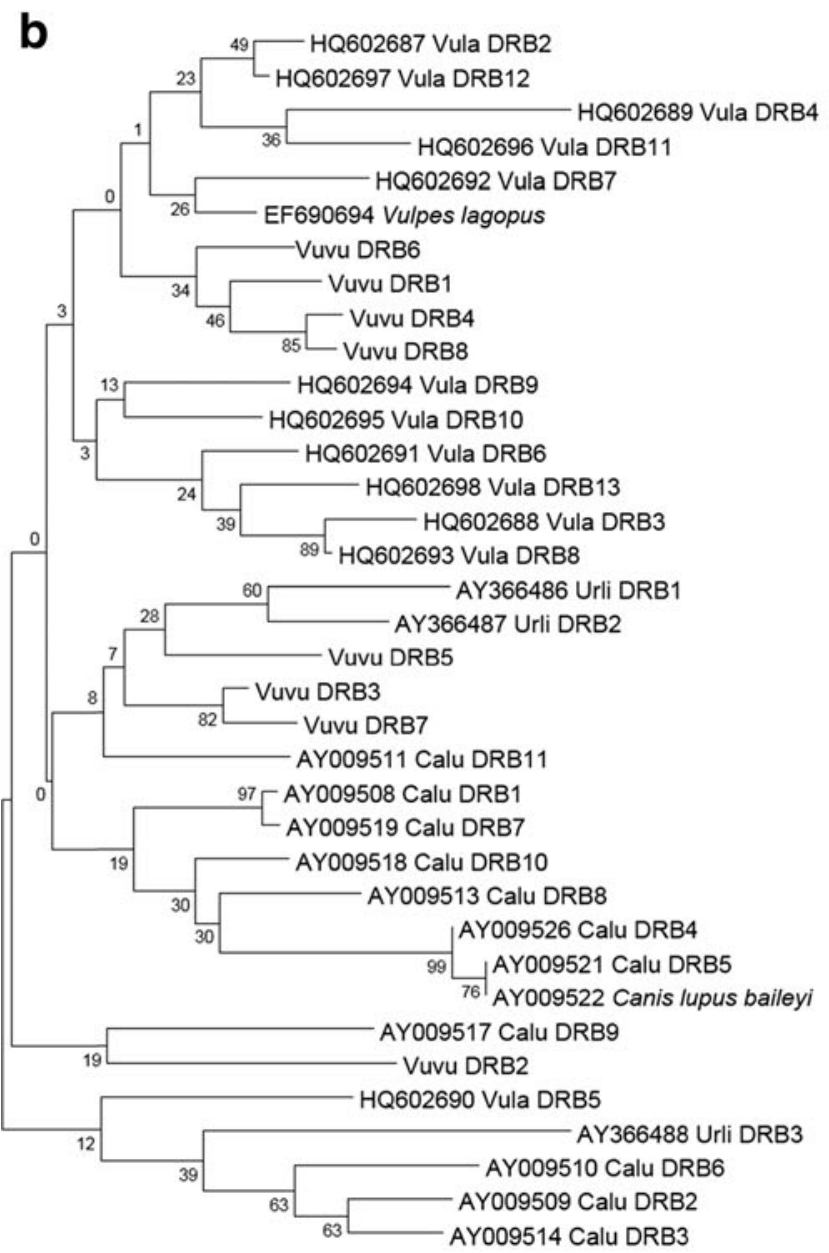

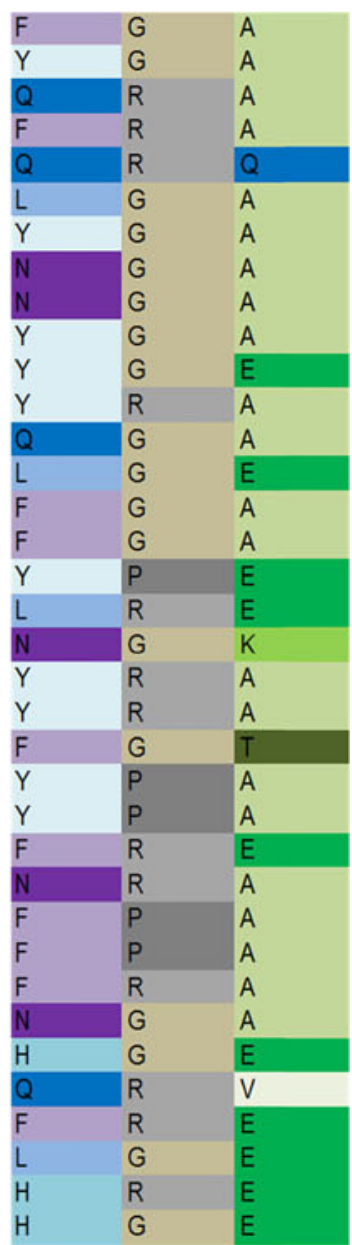


Table 2 Measures of DRB1 diversity and $Z$ tests of neutrality in six Newfoundland island samples of red fox

\begin{tabular}{|c|c|c|c|c|c|c|c|c|c|}
\hline \multirow[t]{2}{*}{ Locality } & \multirow[t]{2}{*}{$2 \mathrm{~N}$} & \multicolumn{6}{|c|}{ Measure of diversity } & \multicolumn{2}{|c|}{$\mathrm{Z}$ test of neutrality } \\
\hline & & $N_{\mathrm{A}}$ & $H_{\mathrm{O}}$ & $H_{\mathrm{E}}$ & $\pi$ & $\mathrm{dN}$ & $\mathrm{dS}$ & $Z$ & $P$ \\
\hline NOP & 10 & 5 & 1.00 & 0.78 & 0.061 & 0.070 & 0.032 & 2.5 & 0.013 \\
\hline WEC & 8 & 4 & 0.50 & 0.56 & 0.030 & 0.036 & 0.013 & 2.2 & 0.030 \\
\hline NEC & 10 & 6 & 0.80 & 0.76 & 0.049 & 0.059 & 0.019 & 2.9 & 0.004 \\
\hline CEN & 8 & 5 & 0.75 & 0.69 & 0.057 & 0.066 & 0.029 & 2.3 & 0.022 \\
\hline $\mathrm{SOC}$ & 10 & 6 & 0.80 & 0.80 & 0.058 & 0.069 & 0.025 & 3.1 & 0.003 \\
\hline AVP & 10 & 5 & 1.00 & 0.78 & 0.059 & 0.069 & 0.029 & 2.6 & 0.011 \\
\hline All & 56 & 8 & 0.82 & 0.82 & 0.054 & 0.064 & 0.026 & 2.7 & 0.007 \\
\hline
\end{tabular}

Locality abbreviations are given in Table 1

$N$ sample size, $H_{\mathrm{O}}$ observed heterozygosity, $H_{\mathrm{E}}$ expected heterozygosity, $\pi$ nucleotide diversity, $d N$ nonsynonymous substitution rate, $d S$ synonymous substitution rate
(Fig. 3b), with particular amino acid variable within species but shared between alleles of different species. For example, at site 30, all four amino acids found in the red fox are also found in alleles from the other species. Notably, three of the four sites under positive selection $(30,64$, and 67$)$ were identified by Brown et al. (1993; sites 37, 71, and 74 in the reference) as antigen binding sites. These patterns provide evidence of trans-species polymorphism at the amino acid level, consistent with selection over the history of species.

The neighbor-joining phylogenies among canid DRB1 alleles are shown in Fig. 3 for the two groups: all Vulpes (Fig. 3a) and Vulpes, Urocyon, and C. lupus (Fig. 3b). In the Vulpes phylogeny (Fig. 3a), although alleles cluster into three groups - a lagopus only group, a vulpes only group, and a mixed group - few nodes are supported by bootstrap values $>50$ and those that do cluster pairs of terminal branches rather than the main clusters. Similarly in the comprehensive phylogeny (Fig. 3b), there are species-specific and non-speciesspecific clusters but little bootstrap support at basal nodes. Thus, although the Vulpes-only phylogeny and the comprehensive phylogeny are consistent with trans-species polymorphism, the lack of bootstrap support prevents any strong conclusion in this respect. Nonetheless, phylogenetic

Table 3 Measures of pairwise population differentiation among red fox sampling localities, inferred from DRB1 sequences

\begin{tabular}{lllllll}
\hline & NOP & WEC & NEC & CEN & SOC & AVP \\
\hline NOP & & 0.095 & -0.026 & -0.026 & -0.058 & -0.047 \\
WEC & 0.107 & & $\mathbf{0 . 1 3 1}$ & $\mathbf{0 . 1 7 6}$ & 0.022 & 0.070 \\
NEC & -0.031 & $\mathbf{0 . 1 4 5}$ & & 0.010 & -0.007 & -0.026 \\
CEN & -0.034 & $\mathbf{0 . 1 9 8}$ & 0.011 & & 0.013 & -0.051 \\
SOC & -0.070 & 0.023 & -0.008 & 0.013 & & -0.038 \\
AVP & -0.057 & 0.080 & -0.031 & -0.067 & -0.045 & \\
\hline
\end{tabular}

Below diagonal: $F_{\mathrm{ST}}$; above diagonal: $d_{\mathrm{A}}$. Values in bold are significant at $\alpha=0.05$. Locality abbreviations are as in Table 1 relationships among Canis DRB1 alleles presented in earlier studies showed distinct lineages of alleles in different species supported by fairly high bootstrap estimates (Hedrick et al. 2000). This along with the evidence for shared positively selected sites found here supports balancing selection at a species timescale.

As is the case generally in mammals, patterns of DRB1 allelic diversity in Newfoundland red foxes are consistent with balancing selection in the long term, over the history of species. Evidence for this is the greater number of alleles (eight in $2 \mathrm{~N}=56$ alleles cf. seven haplotypes among $159 \mathrm{CR}$ sequences; Langille et al. 2014) and order of magnitude higher nucleotide divergence at the DRB1 locus in Newfoundland red foxes in comparison with the mitochondrial CR. Most of the sequence variants are associated with amino acid differences; hence, codon-based $Z$ tests for departures from neutrality are strongly supportive of positive selection at a sequence level in the long term. The possibility of trans-species polymorphism in the phylogeny of DRB1 alleles among Vulpes and more broadly among canids suggests a role for balancing selection transcending species divergences in contributing to allelic diversity in Newfoundland red foxes.

The excess of nonsynonymous to synonymous substitutions that exists between alleles is among the most longstanding and powerful lines of evidence in favor of balancing selection at the MHC and is well documented in the peptidebinding regions of the DRB1 in a wide array of vertebrates (Garrigan and Hedrick 2003). Because this signal takes both a long time to develop and to degrade, it is difficult to make inferences about the timescale of this selection other than that it occurred in the distant past (Garrigan and Hedrick 2003). High amino acid sequence diversity of the MHC class II DRB1 polypeptide provides variation in antigen binding, providing a basis for selective pressure through the ability to resist a wider variety of parasites and pathogens (Edwards and Potts 1996). There is evidence that this is ongoing within $V$. vulpes. Indeed, the $\mathrm{dN} / \mathrm{dS}$ ratio among red fox alleles is higher than 
among all canid alleles ( 2.4 vs $\sim 1.7)$, consistent with continuing selective pressure since species divergence, and we observe a new amino acid variant in red foxes at a site under positive selection among Vulpes.

\section{DRB1 diversity in red foxes compared with other canids}

We can now compare DRB1 diversity in Newfoundland island red foxes to island populations of two other fox species: the island fox (Uricyon littoralis) in the Channel Islands, with a colonization history timescale of $800-16,000$ years ago, and the Arctic fox (V. lagopus) in the Commander Islands, isolated from the mainland since the Pleistocene. Among the Channel Islands, expected heterozygosity ranges from 0 to 0.36 for a set of populations of effective populations sizes of 163-984 and levels of neutral microsatellite genetic variation on the order of $0-0.36$. Notably, the San Nicolas Island population (U. littoralis dickeyi) showed both the lowest microsatellite and the highest DRB diversity, attributed by the authors to a severe bottleneck accompanied by strong selection (Aguilar et al. 2004). Hedrick (2004) cautioned against overinterpretation of the role of selection and suggested a combination of selective and non-selective forces to explain the patterns described by Aguilar et al. By way of contrast, in the Commander Islands, Ploshnitsa et al. (2011) observed a heterozygosity of $\sim 0.73$ in the isolated Bering population but zero in the Mednyi population which suffered a strong bottleneck due to an outbreak of mange in the 1970s. These authors concluded that even strong balancing selection was not enough to overcome a severe bottleneck. The heterozygosity reported here (Table 2) for the Newfoundland island population of red foxes is the highest of any of these island populations, consistent with the ongoing gene flow it experiences with the mainland, as well as a much larger effective population size, and no recent bottlenecks or expansions (Langille et al. 2014).

Investigation of DRB1 alleles from Canis, Vulpes, and Urocyon species allows some general observations to be made. First, the $\mathrm{dN} / \mathrm{dS}$ ratios are similar among the three genera ( $\sim 2.4$ in Vulpes, $\sim 2.2$ in Canis, and $\sim 1.7$ in Urocyon [only three alleles available]). Notably, the $\mathrm{dN}$ measures are virtually identical among taxa, and the differences in ratios are explained by dS differences. This suggests that over the long term, there may be similar levels of selective pressure in each of these taxa. Second, while species or genera tend to group together loosely phylogenetically, the inclusion of all genera illuminates the tendency of alleles to be shared past species and even genus barriers, although this is not well supported by bootstrap analysis, probably due to the length of the sequence analyzed. We also note that unlike in Canis, deeper divergences within the Vulpes only phylogeny are not well supported by bootstrap values. This may be due to longer divergences times among species in the two genera.

\section{Conclusions and future directions}

The signature of positive and balancing selection in red foxes in Newfoundland is consistent with the known functional significance of the genes of the MHC and particularly the DRB1 in numerous studies across vertebrates. Species which exhibit low or no detectable polymorphism in MHC genes might suffer from increased susceptibility to disease, whether enzootic or epizootic (Hedrick, 1994). Here, we also provide evidence that this may be not just in the long term but over the history of the population, as may be expected when establishing a new environment. Whether the new variants observed here are unique to this population remains to be seen.

As suggested by Castillo et al. (2010) for raccoons, the available DRB1 locus data will also allow continued spatial and temporal analysis of the immunogenetic response of red foxes to infection and the influences of diseases on molecular evolution of this important class of loci and about the role of selection and exposure to parasites in the maintenance of genetic diversity of the immune system. There is no evidence for a relationship between parasitic exposure and DRB1 diversity or particular alleles in the Newfoundland population of red foxes, nor of selection in the current generation, establishing a baseline for monitoring future genetic changes in this potentially vulnerable species. As noted by Sommer (2005), the MHC class II region regions are closely linked in mammals, so the pattern observed for the DRB1 locus should be a good indicator of the genetic variation in other class II genes. Going forward, our long-term goals are to broaden our examination to include other genes, other species, and particular references to individuals of specific disease status.

Acknowledgments We thank Jillian Janes and Lorielle Pevie for excellent laboratory assistance. Financial assistance for this research was provided by an NSERC Discovery Grant and by the Dean of Science, Memorial University of Newfoundland. We are very grateful to Cathy Keane (Animal Health Division) and Mac Pitcher (Salmonier Nature Park) for their efforts in coordinating sample collection. We are grateful to personnel at the $\mathrm{GaP}$ Facility, CREAIT Network, of Memorial University for assistance with Sanger sequencing and helpful discussions. We thank two anonymous reviewers for their detailed and constructive suggestions.

Open Access This article is distributed under the terms of the Creative Commons Attribution 4.0 International License (http:// creativecommons.org/licenses/by/4.0/), which permits unrestricted use, distribution, and reproduction in any medium, provided you give appropriate credit to the original author(s) and the source, provide a link to the Creative Commons license, and indicate if changes were made.

\section{References}

Aguilar A, Roemar G, Debenham S, Binns M, Garcelon D, Wayne RK (2004) High MHC diversity maintained by balancing selection in an otherwise genetically monomorphic mammal. PNAS 101(10): $3490-3494$ 
Babik W, Durka W, Radwan J (2005) Sequence diversity of the MHC DRB gene in the Eurasian beaver (Castor fiber). Mol Ecol 14(14):4249-4257

Biedrzycka A, Kloch A, Buczek M, Radwan J (2011) Major histocompatibility complex DRB genes and blood parasite loads in fragmented populations of the spotted suslik Spermophilus suslicus. Mammalian Biol 76(6):672-677

Bowen L, Aldridge BM, Gulland F, Van Bonn W, DeLong R et al (2004) Class II multiformity generated by variable MHC-DRB region configuration configurations in the California sea lion (Zalophus californianus). Immunogenetics 56(1):12-27

Brown JH, Jardetzky TS, Gorga JC, Stern LJ, Urban RG, Strominger JL, Wiley DC (1993) Three-dimensional structure of the human class II histocompatibility antigen HLA-DR1. Nature 364(6432):33-39

Buitkamp J, Filmether P, Stear MJ, Epplen JT (1996) Class I and class II major histocompatibility complex alleles are associated with faecal egg counts following natural, predominantly Ostertagia circumcincta infection. Parasitol Res 82(8):693-696

Castillo S, Srithayakumar V, Meunier V, Kyle CJ (2010) Characterization of major histocompatibility complex (MHC) DRB exon 2 and DRA exon 3 fragments in a primary terrestrial rabies vector (Procyon lotor). PLoS ONE 5(8), e12066

Conboy GA (2011) Canine angiostrongylosis: the French heartworm: an emerging threat in North America. Vet Parasitol 176(4):382-389

Dodds D (1983) Terrestrial mammals. In: South GR (ed) Biogeography and ecology of the island of Newfoundland. Dr. W. Junk Publishers, The Hague, pp 509-550

Edwards SV, Potts WK (1996) Polymorphism of genes in the major histocompatibility complex (MHC): implications for conservation genetics. In: Eds Smith TB, Wayne RK (eds) Molecular genetic approaches in conservation. Oxford University Press, New York, pp 214-237

Excoffier L, Laval G, Schneider S (2007) Arlequin ver. 3.0: an integrated software package for population genetics data analysis. Evol Bioinformatics Online 1:47-50

Garrigan D, Hedrick PW (2003) Perspective: detecting adaptive molecular polymorphism: lessons from the MHC. Evolution 57(8):1707-1722

Grimholt U, Larsen S, Nordmo R, Midtlyng P, Kjoeglum S, Storset A, Saebo S, Stet RJM (2003) MHC polymorphism and disease resistance in Atlantic salmon (Salmo salar); facing pathogens with single expressed major histocompatibility class I and class II loci. Immunogenetics 55(4):210-219

Hedrick PW (1994) Evolutionary genetics of the major histocompatibility complex. Am Nat 143(6):945-964

Hedrick PW (2004) Evolutionary genomics: foxy MHC selection story. Heredity 93(3):237-238

Hedrick PW, Thomson G (1983) Evidence for balancing selection at HLA. Genetics 104(3):449-456

Hedrick PW, Lee RN, Parker KM (2000) Major histocompatibility complex (MHC) variation in the endangered Mexican wolf and related canids. Heredity 85(6):617-624

Hedrick PW, Lee RN, Garrigan D (2002) Major histocompatibility complex variation in red wolves: evidence for common ancestry with coyotes and balancing selection. Mol Ecol 11(10):1905-1913

Hedrick PW, Lee RN, Buchanan C (2003) Canine parvovirus enteritis, canine distemper, and major histocompatibility complex genetic variation in Mexican wolves. J Wildl Dis 39(4):909-913

Huchard E, Cowlishaw G, Raymond M, Weill M, Knapp LA (2006) Molecular study of Mhc-DRB in wild chacma baboons reveals high variability and evidence for trans-species inheritance. Immunogenetics 58(10):805-816

Hughes AL, Nei M (1988) Pattern of nucleotide substitution at major histocompatibility complex class I loci reveals over-dominant selection. Nature 335(6186):167-170

Hughes AL, Yeager M (1998) Natural selection at major histocompatibility complex loci of vertebrates. Annu Rev Genet 32:415-435

Jeffery RA, Lankester MW, McGrath MJ, Whitney HG (2004) Angiostrongylus vasorum and Crenosoma vulpis in red foxes (Vulpes vulpes) in Newfoundland, Canada. Can J Zool 82(1):66-74
Klitz W, Thomson G (1987) Disequilibrium pattern analysis. II. Application to Danish HLA A and B locus data. Genetics 116(4):633-643

Kohn MH, Murphy WS, Ostrander EA, Wayne RK (2006) Genomics and conservation genetics. Trends Ecol Evol 21(11):629-637

Lancaster A, Nelson MP, Single RM, Meyer D, Thomson G (2003) PyPop: a software framework for population genomics: analyzing large-scale multi-locus genotype data. In: Altman RB et al (eds) Pacific symposium on biocomputing, vol 8., pp 514-525

Langille BL, O'Leary KE, Whitney HG, Marshall HD (2014) Mitochondrial control region diversity and phylogeography of insular Newfoundland red foxes. J Mammal 95(4):772-780

Larivière S, Pasitschniak-Arts M (1996) Vulpes vulpes. Mamm Species 537:1-11

Lively CM, de Roode JC, Duffy MA, Graham AL, Koskella B (2014) Interesting open questions in disease ecology and evolution. Am Nat 184(Supplement):S1-S8

Marsden CD, Mable BK, Woodroffe R, Rasmussen GSA, Cleaveland S, McNutt JW, Emmanuel M, Thomas R, Kennedy LJ (2009) Highly endangered African wild dogs (Lycaon pictus) lack variation at the major histocompatibility complex. J Hered 100(Supplement 1):S54-S65

McClelland EE, Granger DL, Potts WK (2003) Major histocompatibility complex-dependent susceptibility to Cryptococcus neoformans in mice. Infect Immun 71(8):4815-4817

McGrath M, Dredge M, Curran R, Reynolds J (2010) Harvest providing valuable insight into coyote ecology. Department of Environment and Conservation, Natural Heritage Branch 6, 1-2

Nei M, Li WH (1979) Mathematical model for studying genetic variation in terms of restriction endonucleases. PNAS 76(10):5269-5273

Paterson S, Wilson K, Pemberton JM (1998) Major histocompatibility complex variation associated with juvenile survival and parasite resistance in a large unmanaged ungulate population (Ovis aries L.). Evolution 95(7):3714-3719

Ploshnitsa AI, Goltsman ME, Macdonald DW, Kennedy LJ, Sommer S (2011) Impact of historical founder effects and a recent bottleneck on MHC variability in commander Arctic foxes. Ecol Evol 2(1):165-180

Saitoh K, Chen WJ (2008) Reducing cloning artifacts for recovery of allelic sequences by $\mathrm{T} 7$ endonuclease I cleavage and single reextensions of PCR products - a benchmark. Gene 423(1):92-95

Satta Y, O'hUigin C, Takahata N, Klein J (1994) Intensity of natural selection at the major histocompatibility complex loci. Proc Natl Acad Sci U S A 91(15):7184-7188

Sommer S (2005) The importance of immune gene variability (MHC) in evolutionary ecology and conservation. Front Zool 2(16) doi:10. 1186/1742-9994-2-16

South GR (1983) Introduction. In: South GR (ed) Biogeography and ecology of the island of Newfoundland. Dr. W. Junk Publishers, The Hague, pp 1-4

Takahata N (1990) A simple genealogical structure of strongly balanced allelic lines and trans-species evolution of polymorphism. Proc Natl Acad Sci U S A 87(7):2419-2423

Tamura K, Stecher G, Peterson D, Filipski A, Kumar S (2013) MEGA 6: molecular evolutionary genetics analysis version 6.0. Mol Biol Evol 30(12):2725-2729

Tsai KL, Starr-Moss AN, Venkataraman GM, Robinson C, Kennedy LJ, Steiner JM, Clark LA (2013) Alleles of the major histocompatibility complex play a role in the pathogenesis of pancreatic acinar atrophy in dogs. Immunogenetics 65(7):501-509

van Oosterhout C (2009) A new theory of MHC evolution: beyond selection on the immune genes. Proc R Soc B 276(1657):657-665

Wassom DL, Brooks BO, Babisch JG, David CS (1983) A gene mapping between the $\mathrm{S}$ and $\mathrm{D}$ regions of the $\mathrm{H}-2$ complex influences resistance to Trichinella spiralis infections in mice. J Immunogenet 10(5):371-378

Yasukochi Y, Satta Y (2013) Current perspectives on the intensity of natural selection of MHC loci. Immunogenetics 65(6):479-483 